\title{
SOME CLASSES \\ OF INDECOMPOSABLE VARIETIES OF GROUPS
}

\author{
JOHN COSSEY 1
}

(Received 14 August 1967)

\section{Introduction}

A variety of groups is an equationally defined class of groups: equivalently, it is a class of groups closed under the operations of taking cartesian products, subgroups, and quotient groups. If $\mathfrak{U}$ and $\mathfrak{B}$ are varieties, then $\mathfrak{U} \mathfrak{B}$ is the class of all groups $G$ with a normal subgroup $N$ in $\mathfrak{U}$ such that $G / N$ is in $\mathfrak{B} ; \mathfrak{U} \mathfrak{B}$ is a variety, called the product of $\mathfrak{U}$ and $\mathfrak{B}$. We denote by (5) the variety generated by the unit group, and by $\mathscr{D}$ the variety of all groups. We say that a variety $\mathfrak{B}$ is indecomposable if $\mathfrak{B} \neq \mathfrak{E}, \mathfrak{B} \neq \mathbb{D}$, and $\mathfrak{B}$ cannot be written as a product $\mathfrak{X Y}$, with both $\mathfrak{X} \neq \mathbb{E}$ and $\mathfrak{Y} \neq \mathbb{E}$. One of the basic results in the theory of varieties of groups is that the set of varieties, excluding $\mathfrak{D}$, and with multiplication of varieties as above, is a free semigroup, freely generated by the indecomposable varieties. Thus one would like to be able to decide whether a given variety is indecomposable or not. In connection with this question, Hanna Neumann raises the following problem (as part of Problem 7 in her book [7]):

Problem 1. If $\mathfrak{U} \$ \mathfrak{B}$, and $\mathfrak{B} \$ \mathfrak{U}$, prove that $[\mathfrak{U}, \mathfrak{B}]$ is indecomposable unless both $\mathfrak{U}$ and $\mathfrak{B}$ have a common non-trivial right hand factor.

(If $G$ is an arbitrary group, and $\mathfrak{U}$ any variety, denote by $U(G)$ the intersection of all normal subgroups of $G$ whose quotient group is in $\mathfrak{l}$ : clearly $G / U(G) \in \mathfrak{U}$, and $U(G)$ is the smallest normal subgroup of $G$ with this property. Then $[\mathfrak{U}, \mathfrak{B}]$ is the variety of all groups $G$ for which $U(G)$ and $V(G)$ centralize each other.)

In this paper, we solve Problem 1 for a class of varieties which includes many of the well known varieties. To state our theorem, we need some notation. Following Philip Hall, we denote by $\mathscr{F}$ the class of all finite groups, and by $\mathscr{N}$ the class of all nilpotent groups: then $\mathscr{F} \mathscr{N}$ denotes the class of all groups which have a finite normal subgroup whose quotient group is nilpotent. The main result of this paper is then

THEOREM 1. Suppose that $\mathfrak{U}$ and $\mathfrak{B}$ are varieties each of which can be

1 The author is a Fulbright-Hays scholar. 
generated by a group in $\mathscr{F} \mathscr{N}$. Then if $\mathfrak{U} \neq \mathfrak{B}$, [uU, $\mathfrak{Y}]$ is indecomposable unless both $\mathfrak{U}$ and $\mathfrak{B}$ have a common non-trivial right hand factor.

Theorem 1 has a couple of special cases that are worth noting. Firstly, if both $\mathfrak{U}$ and $\mathfrak{B}$ are nilpotent, then they are indecomposable ([7] Theorem 24.34), and hence we have

CoRollary 1. If both $\mathfrak{U}$ and $\mathfrak{B}$ are nilpotent, and $\mathfrak{U} \neq \mathfrak{B}$, then $[\mathfrak{U}, \mathfrak{B}]$ is indecomposable.

Another special case, which is a partial result on the way to Theorem 1, is

THEOREM 2. If either $\mathfrak{U}$ or $\mathfrak{B}$ cannot be generated by a finite group (but each can be generated by a group in $\mathscr{F} \mathcal{N})$, then $[\mathfrak{U}, \mathfrak{B}]$ is indecomposable.

\section{Notation and preliminaries}

The main tool used in the proof of Theorem 1 is the (standard) wreath product of groups, and we will assume familiarity with the construction and basic properties of this wreath product: for a detailed description, see [7] Section 2.2. We will also adopt the notation used there.

Other notation is in general standard. We denote the fact that $H$ is a subgroup of $G$ by $H \leqq G$ : if $H$ is a proper subgroup, by $H<G$. When $H$ is normal in $G$, we put $H \unlhd G$. As usual, $[x, y]=x^{-1} y^{-1} x y$ : if $H \leqq G$, $K \leqq G$, then $[H, K]$ is the subgroup of $G$ generated by all $[h, k], h \in H, k \in K$. If $S$ is a subset of $G$, the centraliser of $S$ in $G$ is denoted by $C_{G}(S)$. If $S$ is a subset of $G$, the subgroup of $G$ generated by $S$ is denoted by $\langle S\rangle$ : if $S=\left\{x_{1}, \cdots, x_{n}\right\},\langle S\rangle=\left\langle x_{1}, \cdots, x_{n}\right\rangle$. The centre of a group $G$ is denoted by $\zeta(G)$.

If $\mathfrak{U}, \mathfrak{B}$ are varieties, then $\mathfrak{U} \leqq \mathfrak{B}$ means that $\mathfrak{U}$ is a subvariety of $\mathfrak{B}: \mathfrak{U}<\mathfrak{B}$ means that $\mathfrak{U}$ is a proper subvariety of $\mathfrak{B}$. The union $\mathfrak{U} \cup \mathfrak{B}$ of two varieties is the variety generated by the union of the classes $\mathfrak{U}$ and $\mathfrak{B}$ : the intersection $\mathfrak{U} \cap \mathfrak{B}$ is just the class of all groups $G$ such that $G \in \mathfrak{U}$ and $G \in \mathfrak{B}$. If $\mathbb{E}$ is a class of groups, then var $\mathbb{E}$ denotes the variety generated by (E: if $\mathbb{E}$ consists of a single group $G$, var $G=\operatorname{var} \mathbb{E}$. If $G$ is finite, we call $\operatorname{var} G$ a Cross variety. If $\mathfrak{U}$ is locally finite, there is a smallest integer $e$ such that the exponent of any group in $\mathfrak{U}$ divides $e$ : we call $e$ the exponent of $\mathfrak{u} . \mathfrak{A}_{n}$ will denote the variety of all abelian groups of exponent dividing $n: \mathfrak{A}$ denotes the variety of all abelian groups. If $p$ is a prime, $\mathfrak{Q}_{p}$ denotes the variety of all groups which are central extensions of elementary abelian $p$-groups by elementary abelian $p$-groups and are of exponent dividing $p^{2}$.

If $G$ is a finite group, and $1 \leqq N \leqq H \leqq G$, we say that $H / N$ is a factor of $G$ : if either $\mathrm{l} \neq N$ or $H \neq G$, we say that $H / N$ is a proper factor. If $G$ is not in the variety generated by its proper factors, we say that $G$ 
is critical. If $G$ is critical, then $G$ has a unique minimal normal subgroup, called the monolith of $G$, and denoted by $\sigma G$.

Next, some observations about varieties which can be generated by a group in $\mathscr{F} \mathscr{N}$. In [4], Philip Hall showed that $\mathscr{F} \mathscr{N}$ was also the class of all groups in which some finite term of the upper central series was of finite index: it is this characterisation of the class $\mathscr{F} \mathscr{N}$ that seems more useful from the viewpoint of varieties. Groups in $\mathscr{F} \mathscr{N}$ are closely related to both finite and nilpotent groups, and in fact the varieties they generate enjoy many of the pleasant properties of nilpotent and Cross varieties.

LEMma 2.1. Suppose that $\mathfrak{U}$ and $\mathfrak{B}$ are varieties which can be generated by a group in $\mathscr{F} \mathscr{N}$. Then we have

(a) $\mathfrak{U} \cup \mathfrak{B}$ can also be generated by a group in $\mathscr{F} \mathscr{N}$.

(b) $\mathfrak{U}$ and all its subvarieties are generated by finitely generated groups.

(c) Every finitely generated group in $\mathfrak{U}$ is in $\mathscr{F} \mathscr{N}$.

(d) $\mathfrak{u}$ is generated by its finite groups.

(e) There is a bound on the class of nilpotent groups in $\mathfrak{U}$.

(f) There is a bound on the minimal number of generators of chief factors of finite groups in $\mathfrak{u}$.

These results are either easy to prove or are contained in [2].

We often need the following fact that, though well known, does not seem to be readily available in the literature. Denote by $C(p, q)$ the critical group with an elementary abelian normal $p$-subgroup, with quotient group of order $q, p, q$ distinct primes: we have $\operatorname{var} C(p, q)=\mathfrak{A}_{p} \mathfrak{A}_{a}$.

LEMMA 2.2. If $\mathfrak{U}$ is a variety which contains non-abelian finite groups, then it contains either a non-abelian group of order $p^{3}$ for some prime $p$, or a non-nilpotent group $C(p, q), p, q$ distinct primes. If $\mathfrak{u}$ contains nonnilpotent finite groups, it contains a $C(p, q)$ for distinct primes $p, q$.

Proof. We give a sketch of the proof. There are two cases to consider.

If every non-abelian finite group in $\mathfrak{U}$ is nilpotent, $\mathfrak{U}$ contains finite non-abelian groups which are nilpotent of class two. Let $G$ be a non-abelian nilpotent finite group in $U$ such that every proper factor of $G$ is abelian. Firstly, $G$ will be a $p$-group for some prime $p$, and if $x, y \in G$ are such that $[x, y] \neq 1$, then we must have $\langle x, y\rangle=G$. Also, every proper homomorphic image of $G$ is abelian, and so, by Theorem 5 of M. F. Newman [9], $G$ is either non-abelian of order $p^{3}$ and exponent $p$, in which case we are finished, or $G$ is isomorphic to a group of the form

$$
\left\{a, b, z: a^{p}=b^{p}=z, z^{p^{n-1}}=[a, b],[a, b]^{p}=1\right\}, n \geqq 1 .
$$

In this case, $\operatorname{var} G$ is defined by the laws $x^{p^{n+1}}=1,[x, y]^{p}=1,[x, y, t]=1$, 
and any non-abelian group of order $p^{3}$ satisfies these laws, and so is contained in var $G$ and hence in $\mathfrak{H}$.

Now, suppose that $\mathfrak{U}$ contains a non-nilpotent finite group. Let $G$ be a non-nilpotent finite group of minimal order in $\mathfrak{U}$. Then every subgroup of $G$ is nilpotent, and so $G$ is soluble (L. Redei [11]). Let $N$ be a minimal normal subgroup of $G: N$ is an elementary abelian $p$-group for some prime $p$. Also, $N$ cannot be in the centre of $G$, for $G / N$ is nilpotent. It follows that there is an element $x \in G$ of prime order $q$ such that $x \notin C_{G}(N)$. But then $\langle N, x\rangle=N\langle x\rangle$ is non-nilpotent, and so $G=N\langle x\rangle$. It now follows that $G \cong C(p, q)([10]$ p. 364)

Another trivial but important fact is

Lemma 2.3. For any varieties $\mathfrak{U}, \mathfrak{B}$, we have

$$
[\mathfrak{H}, \mathfrak{B}] \leqq[\mathfrak{U} \cup \mathfrak{B}, \mathfrak{U} \cup \mathfrak{B}]=\mathfrak{A}(\mathfrak{U} \cup \mathfrak{B})
$$

Thus, for $G \in[\mathfrak{U}, \mathfrak{B}]$, there is an abelian normal subgroup $N$ of $G$ such that $G / N \in \mathfrak{U} \cup \mathfrak{B}$.

LEMMA 2.4. If $G$ is a non-abelian finite group, $H$ a finite group, and if $A$ is a maximal abelian normal subgroup of $G \mathrm{wr} H$, then there is a maximal abelian normal subgroup $A_{0}$ of $G$ such that if $B\left(=G^{H}\right)$ is the base group of Gwr $H$,

$$
A=\left\{f \in B: f(h) \in A_{\mathbf{0}}, \text { for all } h \in H\right\} .
$$

Proof. We claim firstly that $A \leqq B$. For suppose that $f h \in A, f \in B$, $h \in H$, and $h \neq 1$. Let $A_{1}=A \cap B$, and for $k \in H$ the epimorphism $\pi_{k}: B \rightarrow G$ be defined by

$$
f \pi_{k}=f(k) \text {. }
$$

Then $A_{1} \pi_{k}$ is abelian: in particular $A_{1} \pi_{1}$ is abelian. Since $G$ is non-abelian, there is an $x \in G$, such that $x \notin A_{1} \pi_{1}$. Define $g \in B$ by $g(1)=x^{-1}, g(k)=1$, $k \neq 1$. Now since both $A$ and $B$ are normal, $[g, f h] \in A \cap B=A_{1}$. Since $h \neq 1, \quad\left(f^{-1} g f\right)^{h}(1)=f^{-1} g f\left(h^{-1}\right)=1$, from the definition of $g$, and so $[g, f h](1)=x$ : i.e. $x \in A_{1} \pi_{1}$, a contradiction. Thus $A \leqq B$.

Now, it is easy to check that $A \pi_{h}=A \pi_{1}$ for all $h \in H$. Thus if $A_{0}$ is a maximal abelian normal subgroup of $G$ containing $A \pi_{1}$,

But clearly

$$
A \leqq\left\{f \in B: f(h) \in A_{0}, h \in H\right\} .
$$

$$
\left\{f \in B: f(h) \in A_{0}, h \in H\right\}
$$

is an abelian normal subgroup of $G$ wr $H$, and hence the result follows.

Then we have as a consequence of Lemma 2.4 and Theorems 22.11 and 22.12 of [7] the following lemma. 
Lemma 2.5. If $G$ is a non-abelian finite group, $H$ a finite group, $A$ a maximal abelian normal subgroup of $W=G \mathrm{wr} H, B$ the base group of $W$, then $W / A$ contains a factor isomorphic to $C_{p} \mathrm{wr} H$, where $C_{p}$ is a cyclic group of order $p$, for all primes $p$ dividing $|B| A \mid$.

Finally in this section we prove:

LEMMA 2.6. If $\mathfrak{U}$ and $\mathfrak{B}$ are varieties, such that $\mathfrak{U} \neq \mathfrak{B}$, and both $\mathfrak{U}$ and $\mathfrak{B}$ are generated by their finite groups, then

$$
\mathfrak{U}(\mathfrak{U} \cap \mathfrak{B}) \neq[\mathfrak{U}, \mathfrak{B}] .
$$

Proof. First, we show that it is sufficient to prove the lemma for $\mathfrak{U}<\mathfrak{B}$, and $\mathfrak{B}$ generated by its finite groups. For suppose $\mathfrak{U} \nless \mathfrak{B}$, $\mathfrak{F} \nless \mathfrak{U}$, and $[\mathfrak{U}, \mathfrak{B}]=\mathfrak{A}(\mathfrak{U} \cap \mathfrak{B})$. Then

$$
\begin{aligned}
\mathfrak{U}(\mathfrak{U} \cap \mathfrak{B}) & =[\mathfrak{U} \cap \mathfrak{B}, \mathfrak{U} \cap \mathfrak{B}] \\
& \leqq[\mathfrak{U} \cap \mathfrak{B}, \mathfrak{i}] \\
& \leqq[\mathfrak{U}, \mathfrak{B}] \\
& =A(\mathfrak{U} \cap \mathfrak{B})
\end{aligned}
$$

and so $[\mathfrak{U} \cap \mathfrak{B}, \mathfrak{B}]=\mathfrak{H}((\mathfrak{U} \cap \mathfrak{B}) \cap \mathfrak{B})$.

Now, if $\mathfrak{U}<\mathfrak{B}$, and $\mathfrak{B}$ is generated by its finite groups, then there is a finite group $G$ of minimal order such that $G \in \mathfrak{B}, G \notin \mathfrak{U}$. Then we have that $G$ is critical and $G / \sigma G \in \mathfrak{U}$. If $\sigma G$ is non-abelian, then clearly $G \notin[\mathfrak{U}, \mathfrak{U}]$. Hence $\sigma G$ is abelian: and so - as a minimal normal subgroup of $G$ elementary abelian of exponent $p$ for some prime $p$ : hence $G \in \mathfrak{A}_{p} \mathfrak{u}$. Let $\mathfrak{B}_{1}$ be the variety generated by $G$. Then for some positive integer $n$, if $H$ is the relatively free group of rank $n$ of $\mathfrak{B}_{1}, U(H)$ is a non-cyclic elementary abelian $p$-group. Let $F$ be the absolutely free group of rank $n$,

$$
U / V_{1}(F)=U\left(F / V_{1}(F)\right) .
$$

Then $F / V_{1}(F)$ is a finite group ([8] Theorem 14.2), and so by Schreier's theorem $U$ is an absolutely free group of finite rank. To complete the proof of Lemma 2.6, it is now sufficient to prove:

Lemma 2.7. Let $F$ be an absolutely free group of finite rank, $N$ a normal subgroup of $F$ such that $F / N$ is a non-cyclic elementary abelian p-group for some prime $p$. Then $F /[F, N]$ is non-abelian.

Proof. ${ }^{2}$ With $p$ as in the statement of the lemma, we have $N>Q_{p}(F)$. Put $H=F / Q_{p}(F), \quad M=N / Q_{p}(F)$ : then it is sufficient to prove that $H /[H, M]$ is non-abelian. Observe that since $F$ is of finite rank, $n$ say, $H$ is finite. Now $H / \Phi(H)=M / \Phi(H) \times L / \Phi(H)$, where $\Phi(H)$ is the Frattini

2. This proof was suggested to me by Professor G. Baumslag. 
subgroup of $H$. Let $x_{1} \Phi(H), \cdots, x_{k} \Phi(H)$ be a minimal set of generators for $L / \Phi(H)$, and $y_{1} \Phi(H), \cdots, y_{n-k} \Phi(H)$ be a minimal set of generators for $M / \Phi(H)$. Since $H$ is a finite relatively free group of rank $n$, and $H$ is generated by $\left\{x_{1}, \cdots, x_{k}, y_{1}, \cdots, y_{n-k}\right\}=S$, it is freely generated by $S$. Thus the commutators $\left[x_{i}, x_{j}\right], i \neq j, 1 \leqq i, j \leqq k,\left[x_{i}, y_{j}\right], 1 \leqq i \leqq k, 1 \leqq j \leqq n-k$, $\left[y_{i}, y_{i}\right], i \leqq j, 1 \leqq i, j \leqq n-k$, are all independent. But $[H, M]$ is generated by $\left[x_{i}, y_{j}\right], 1 \leqq i \leqq k, 1 \leqq j \leqq n-k,\left[y_{i}, y_{j}\right], i \neq j, 1 \leqq i, j \leqq n-k$, and since $H / M$ was non-cyclic, $k \geqq 2$. Hence $\left[x_{1}, x_{2}\right] \notin[H, M]$, and Lemma 2.7 is proved.

\section{The proof of Theorem 1}

Suppose that $\mathfrak{U}$ and $\mathfrak{B}$ are varieties which can be generated by a group in $\mathscr{F} \mathscr{N}$ and $\mathfrak{U} \neq \mathfrak{B}$. Then, using Lemmas 2.1 and 2.3 , finitely generated groups in $[\mathfrak{U}, \mathfrak{B}]$ are abelian-by-nilpotent-by-finite, and so, as a consequence of Theorem 1 of P. Hall [5], are residually finite. Hence [U, $\mathfrak{Z}]$ is generated by its finite groups.

The proof is broken up into several steps, which we number consecutively. The first step gives some necessary conditions which varieties $\mathfrak{X}, \mathfrak{Y}$ must satisfy if $[\mathfrak{U}, \mathfrak{B}]$ is to equal $\mathfrak{X} \mathfrak{V}$.

3.1 Suppose that $\mathfrak{X}$ and $\mathfrak{Y}$ are varieties: then $[\mathfrak{U}, \mathfrak{B}] \neq \mathfrak{X Y}$ if any of the following conditions hold:

(a) $\mathfrak{X}$ is abelian,

(b) $\mathfrak{X}$ contains a non-abelian group of order $p^{3}$ for some prime $p$, and $\mathfrak{A}_{p} \leqq \mathfrak{V}$,

(c) $\mathfrak{A}_{p} \mathfrak{H}_{q} \leqq \mathfrak{X}, \mathfrak{U}_{q} \leqq \mathfrak{Y}$, for distinct primes $p, q$,

(d) $\mathfrak{X}$ contains a non-abelian finite simple group $G$, and for some prime $p$ dividing $|G|, \mathfrak{A}_{p} \leqq \mathfrak{Y}$.

Proof. (a) Suppose that $\mathfrak{X}$ is abelian and $[\mathfrak{U}, \mathfrak{B}]=\mathfrak{X Y}$. Then $\mathfrak{Y} \leqq \mathfrak{U} \cap \mathfrak{V}$ ([7] Theorem 24.31), and so

$$
\begin{aligned}
{[\mathfrak{U}, \mathfrak{B}] } & =\mathfrak{X} \mathfrak{Y} \\
& \leqq \mathfrak{H}(\mathfrak{U} \cap \mathfrak{V}) \\
& \leqq[\mathfrak{U}, \mathfrak{B}],
\end{aligned}
$$

giving $[\mathfrak{U}, \mathfrak{B}]=\mathfrak{H}(\mathfrak{U} \cap \mathfrak{B})$, contradicting Lemma 2.6.

(b) Suppose $[\mathfrak{U}, \mathfrak{B}]=\mathfrak{X} \mathfrak{Y}$, and that $G \in \mathfrak{X}$ is a non-abelian group of order $p^{3}$, and $C_{p}$ is a cyclic group of order $p$. Then the set

$$
\left\{G \operatorname{wr} C_{p}^{n}: n=.1,2, \cdots\right\} \subseteq \mathfrak{X Y} .
$$

But then, applying Lemma 2.5, 


$$
\left\{C_{p} \text { wr } C_{p}^{n}: n=1,2, \cdots\right\} \subseteq \mathfrak{U} \cup \mathfrak{B} .
$$

But $C_{p}$ wr $C_{p}^{n}$ is nilpotent of class $n(p-1)+1$ ([6] Theorem 5.1), and so $\mathfrak{U} \cup \mathfrak{B}$ contains nilpotent groups of arbitrarily large class, contradicting Lemma 2.1 (e).

(c) Again, suppose $[\mathfrak{U}, \mathfrak{B}]=\mathfrak{X Y}$, and let $C(p, q) \in \mathfrak{X}, C_{q} \in \mathfrak{Y}$, for distinct primes $p, q$. Then the set $\left\{C(p, q)\right.$ wr $\left.C_{q}^{n}: n=1,2, \cdots\right\} \subseteq \mathfrak{X Y}$. Now $C(p, q)$ has a unique maximal abelian normal subgroup, which has index $q$ : hence we may conclude from Lemma 2.5 that

$$
\left\{C_{q} \text { wr } C_{q}^{n}: n=1,2, \cdots\right\} \subseteq \mathfrak{U} \cup \mathfrak{B},
$$

again giving a contradiction.

(d) If $G \in \mathfrak{X}$ is a non-abelian finite simple group, $p$ a prime dividing $|G|$ such that $C_{p} \in \mathfrak{Y}$, then $\left\{G\right.$ wr $\left.C_{p}^{n}: n=1,2, \cdots\right\} \subseteq \mathfrak{X Y}$. But $G$ wr $C_{p}^{n}$ has no non-unit abelian normal subgroups, and so, using Lemma 2.5 again,

$$
\left\{C_{p} \text { wr } C_{p}^{n}: n=1,2, \cdots\right\} \subseteq \mathfrak{U} \cup \mathfrak{B}
$$

if $[\mathfrak{U}, \mathfrak{B}]=\mathfrak{X Y}$ : again giving a contradiction.

Now suppose that $[\mathfrak{U}, \mathfrak{B}]$ is decomposable: that is $[\mathfrak{U}, \mathfrak{B}]=\mathfrak{X Y}$ for some $\mathfrak{X}, \mathfrak{Y}$. Then we have that $\mathfrak{X}$ is non-abelian, and $\mathfrak{Y} \leqq \mathfrak{U} \cap \mathfrak{B}$. If $\mathfrak{Y}$ is not locally finite, then $\mathfrak{A} \leqq \mathfrak{V}$, and we see from Lemma 2.2 that $\mathfrak{X}$ and $\mathfrak{Y}$ must satisfy either (b) or (c) of Lemma 3.1, giving a contradiction. Thus $\mathfrak{Y}$ is locally finite: let the exponent of $\mathfrak{Y}$ be $e$.

3.2 Suppose that $G$ is a finite group in $\mathfrak{X}$. Then $G$ has an abelian normal subgroup $N$ such that $(e,|G / N|)=1$, and for some integer $k,|N|$ divides $e^{k}$.

Proof. If $H$ is any subgroup of $G$ for which there is an integer $k$ such that $|H|$ divides $e^{k}$, then $H$ is abelian: for otherwise, we may conclude from Lemma 2.2 that $\mathfrak{X}$ and $\mathfrak{Y}$ satisfy either (b) or (c) of 3.1, a contradiction.

We now use induction on the length of a chief series of $G$. If the length is one, the result is trivial. Suppose now $G$ has a chief series of length $n$, and the result is true for groups in $\mathfrak{X}$ with a chief series of length $n-1$. Let $M$ be a minimal normal subgroup of $G$ : then $G / M$ has a chief series of length $n-1$. Hence $G / M$ has an abelian normal subgroup $N / M$ satisfying the requirements of the lemma. Now, either $|M|=p^{l}$ for some prime $p$ dividing $e$, or $|M|$ is prime to $e$. In the first case, $N$ is abelian, from the first paragraph of the proof, and we are finished. For the second case, $M$ is complemented in $N$, by $L$ say, using the Schur-Zassenhaus theorem: let $C=C_{L}(M)$. If $C \neq L$, then $N$ is not nilpotent, and so by Lemma 2.2 and the assumption on $L$ contains a factor isomorphic to $C(p, q)$, for $p, q$ distinct primes, with $q$ dividing $e$. But then $\mathfrak{A}_{p} \mathfrak{A}_{q} \leqq \mathfrak{X}, \mathfrak{A}_{q} \leqq \mathfrak{Y}$, a contradiction. Hence $C=L$, 
and so $N=M \times L$, and now $L$ has the properties required of ' $N$ ' in the statement 3.2.

We now prove:

\section{$3.3 \mathfrak{Y}$ is abelian.}

Proof. Since $\mathfrak{X}$ is non-abelian it contains a group $G$ isomorphic to either a non-abelian group of order $p^{3}$ or a $C(q, p), p, q$ distinct primes: it is an immediate consequence of 3.2 that $p$ does not divide $e$. If $\mathfrak{Y}$ is non-abelian it contains a non-abelian group $H$. Then the set

$$
\left\{G \text { wr } H^{n}: n=1,2, \cdots\right\} \subseteq \mathfrak{X Y} .
$$

But then, using Lemma 2.5, we have that

$$
\left\{C_{p} \text { wr } H^{n}: n=1,2, \cdots\right\} \cong \mathfrak{U} \cup \mathfrak{B} .
$$

Now the base group of $C_{p}$ wr $H^{n}$ may be regarded as a vector space over $G F(p)$, the field of $p$ elements, on which $H^{n}$ acts as a group of linear transformations. Since $p$ is prime to $|H|$, the base group is completely reducible, and it contains irreducible components of degree at least $2^{n}$. These irreducible components may then be thought of as chief factors of $C_{p}$ wr $H^{n}$, and so the set $\left\{C_{p}\right.$ wr $\left.H^{n}: n=1,2, \cdots\right\}$ contains groups with chief factors having an arbitrarily large minimal number of generators, contradicting Lemma 2.1 (f). Hence $\mathfrak{Y}$ is abelian.

Now $\mathfrak{U}$ and $\mathfrak{B}$ can be generated by finitely generated groups, $G$ and $H$ say. Now we can choose $G$ and $H$ such that $\mathfrak{Y}=\operatorname{var} G / Y(G)=\operatorname{var} H / Y(H)$. Since $\mathfrak{Y}$ is abelian of finite exponent, $G / Y(G)$ and $H / Y(H)$ are finite. We have further:

3.4 With $G$ and $H$ as above, $Y(G)$ and $Y(H)$ are finite, and

$$
(|G / Y(G)|,|Y(G)|)=(|H / Y(H)|,|Y(H)|)=1 .
$$

Proof. By the symmetry of the situation, it is enough to prove $\mathbf{3 . 4}$ for $G$. Note that if $Y(G)$ is of finite exponent, it is finite. Hence if $Y(G)$ is not finite, then $\mathfrak{A} \leqq \mathfrak{U}$. Let $B$ be a free nilpotent group of class two and rank 2 , generated by $x$ and $y$ : then $B \in[\mathfrak{H}, \mathfrak{B}]$. Also $B_{\mathbf{1}}=\left\langle x^{e}, y^{e}\right\rangle \leqq Y(B)$, and by [1] Theorem 1, $B_{1}$ is also a free nilpotent group of class 2 and rank 2 . If now $p$ is any prime dividing $e, B_{1}$ has non-abelian factors of $p$-power order. But $B_{1} \in \mathfrak{X}$, and so $\mathfrak{X}$ and $\mathfrak{Y}$ satisfy condition (b) of 3.1, a contradiction. Thus $Y(G)$ is finite.

Put $Y(G)=A$ : by 3.2 , there is an abelian normal subgroup $N$ of $A$ such that $(|A| N \mid, e)=1$, and $|N|$ divides $e^{k}$ for some integer $k$. Suppose that $N \neq 1$. There are two cases to consider

(i) $C_{A}(N) \neq A$. Then there is an element $x$ of prime order such that 
$x \notin C_{A}(N)$. Then from Lemma 2.2, $C(p, q) \in \operatorname{var}(N\langle x\rangle) \leqq \mathfrak{X} \cap \mathfrak{U}$ for primes $p, q$ : from the choice of $N\langle\boldsymbol{x}\rangle$, it follows that $p$ divides $e$, and $q$ does not divide $e$. Now (i) divides into two subcases. Firstly, suppose that $V(C(p, q))<C(p, q)$. Then, if $G \in \mathfrak{D}_{p} \mathfrak{H}_{q}, U(G) \leqq \zeta(V(G))$, and so $\mathfrak{D}_{p} A_{q} \leqq[\mathfrak{U}, \mathfrak{B}]=\mathfrak{X Y Y}$. If $|\sigma C(p, q)|=p^{t}$, let $F$ be the free group of $\mathfrak{\Xi}_{p}$ of rank $2 t$ freely generated by $x_{1}, \cdots, x_{t}, y_{1}, \cdots, y_{t}$ : let

$$
\begin{aligned}
& H_{1}=\left\langle x_{1} \zeta(F), \cdots, x_{t} \zeta(F)\right\rangle, \\
& H_{2}=\left\langle y_{1} \zeta(F), \cdots, y_{t} \zeta(F)\right\rangle .
\end{aligned}
$$

On each of $H_{1}, H_{2}$, define the action of $C_{q}$, the cyclic group of order $q$, by the action of $C(p, q) / \sigma C(p, q)$ on $\sigma C(p, q)$, and extend this action to $F$. Let $G=F C_{a}$ : from its definition it follows that $G$ has no quotient groups of $p$-power order, and so we may conclude that $G \in \mathfrak{X}$. But $G$ has a nonabelian Sylow $p$-subgroup, and so $\mathfrak{X}, \mathfrak{Y}$ satisfy (b) of 3.1, a contradiction.

Thus, suppose $V(C(p, q))=C(p, q)$ : it follows that $q$ does not divide the exponent of $\mathfrak{B}$. Further $\mathfrak{A}_{p} \mathfrak{A}_{q} \mathfrak{A}_{p} \leqq \mathfrak{X Y}$, and $\mathfrak{A}_{p} \mathfrak{A}_{q} \mathfrak{A}_{p}$ cannot be generated by a finite group ([7] Theorem 24.62). Since $G$ is finite, $\mathfrak{U}$ can be generated by a finite group, and so there is a critical group $F \in \mathfrak{A}_{p} \mathfrak{A}_{a} \mathfrak{A}_{p}$ such that $\mathbf{1}<M<K \triangleleft F$, with $M$, the unique minimal normal subgroup of $F$, and $F / K$ elementary abelian $p$-groups, and $K / M$ an elementary abelian $q$-group. Further, $F$ has the property that $[M, K] \neq 1$, and $U(F) \geqq$ $M, U(F) \geqq K$. Hence $[U(F), V(F)] \neq 1$, a contradiction.

(ii) $C_{A}(N)=A$. Then $A=N \times N^{*}$, where $N^{*} \cong A / N, N^{*}<G$. Thus $G / A$ and $G / N^{*}$ generate different varieties. We have var $(G / A)=\mathfrak{Y}:$ put $\operatorname{var}\left(G / N^{*}\right)=\mathfrak{V}_{1}$. If $F$ is a free group of finite rank such that $Y(F) / Y_{1}(F)$ is non-cyclic, we have, using Lemma 2.7, that

$$
F>Y(F)>Y_{1}(F)>\left[Y_{1}(F), Y(F)\right],
$$

and $Y(F) /\left[Y_{1}(F), Y(F)\right]$ contains non-abelian factors of $p$-power order for some prime $p$ dividing $e$. But

$$
F /\left[Y_{1}(F), Y(F)\right] \in[\mathfrak{U}, \mathfrak{Y}] \leqq[\mathfrak{U}, \mathfrak{B}]=\mathfrak{X} \mathfrak{Y},
$$

and so $Y(F) /\left[Y_{\mathbf{1}}(F), Y(F)\right] \in \mathfrak{X}$, again giving that $\mathfrak{X}$ and $\mathfrak{Y}$ satisfy (b) of 3.1 , a contradiction.

Thus $N=1$, and 3.4 is proved.

From 3.4 it follows immediately that if either of $G$ or $H$ is infinite, $[\mathfrak{U}, \mathfrak{B}]$ is indecomposable, and so the proof of Theorem 2 is complete.

Now, with $G$ and $H$ as above, put $\mathfrak{U}_{\mathbf{1}}=\operatorname{var}(Y(G)), \mathfrak{B}_{\mathbf{1}}=\operatorname{var}(Y(H))$. Then $\mathfrak{U}_{1}$ and $\mathfrak{B}_{1}$ are locally finite of exponents prime to $e$. The next step is to prove

$3.5\left[\mathfrak{U}_{1}, \mathfrak{B}_{1}\right] \leqq \mathfrak{X}$. 
Proof. Since $\left[\mathfrak{U}_{1}, \mathfrak{B}_{1}\right]$ is generated by its finite groups, if $\left[\mathfrak{U}_{1}, \mathfrak{B}_{1}\right] \nless \mathfrak{X}$, there is a finite group $A$ of minimal order such that $A \in\left[\mathfrak{U}_{1}, \mathfrak{B}_{1}\right], A \notin \mathfrak{X}$. Observe that $\left[\mathfrak{U}_{1}, \mathfrak{B}_{1}\right] \leqq[\mathfrak{U}, \mathfrak{B}]=\mathfrak{X} \mathfrak{Y}$, and so $A \in \mathfrak{X} Y$. If $A$ has order prime to $e$, then $A \in \mathfrak{X}$.

Since $A$ is critical, if $U_{1}(A) \cap V_{1}(A)=1$, then $A$ has order prime to $e$, a contradiction. Now $N=U_{1}(A) \cap V_{1}(A)$ is an abelian normal subgroup of $A$ : since $A$ is critical it must be a $p$-group for some prime $p$, and since $A \notin \mathfrak{X}, p$ must divide $e$. Further, if $N_{1}$ is the subgroup of $N$ generated by the $p^{\text {th }}$ powers of the elements of $N, A / N_{1} \notin \mathfrak{X}$ and $N=\sigma A$. Also, $N \neq A$ since $A \notin \mathfrak{X}$. Since $Y(A) \neq 1, Y(A) \geqq N$. But then $Y(A) / N=Y(A / N)$, and since $A / N \in \mathfrak{U}_{1} \cup \mathfrak{B}_{1}, Y(A / N)=A / N$. Hence $Y(A)=A$, and $A \in \mathfrak{X}$, a contradiction.

$3.6\left[\mathfrak{U}_{1} \mathfrak{Y}, \mathfrak{B}_{1} \mathfrak{Y}\right]=[\mathfrak{U}, \mathfrak{B}]$.

Proof. Using 3.5, [6] Theorem 21.23, and the fact that $\mathfrak{u} \leqq \mathfrak{U}_{1} \mathfrak{V}$, $\mathfrak{B} \leqq \mathfrak{B}_{1} \mathfrak{Y}$, we have

$$
\begin{aligned}
{[\mathfrak{U}, \mathfrak{B}] } & =\mathfrak{X Y} \\
& \geqq\left[\mathfrak{U}_{1}, \mathfrak{B}_{1}\right] \mathfrak{Y} \\
& =\left[\mathfrak{U}_{1} \mathfrak{Y}, \mathfrak{B}_{1} \mathfrak{Y}\right] \\
& \geqq[\mathfrak{U}, \mathfrak{B}],
\end{aligned}
$$

and the result is proved.

We now want to show that in fact $\mathfrak{U}=\mathfrak{U}_{1} \mathfrak{Y}, \mathfrak{B}=\mathfrak{B}_{1} \mathfrak{Y}$. As a step in this direction, we prove:

\section{$3.7 \mathfrak{U}_{1}$ and $\mathfrak{B}_{1}$ are nilpotent.}

Proof. Suppose that $\mathfrak{U}_{1}$ is not nilpotent. Then since $\mathfrak{U}_{1}$ is generated by its finite groups, we may conclude from Lemma 2.2 that $\mathfrak{A}_{p} \mathfrak{A}_{a} \leqq \mathfrak{H}_{1}$ for some distinct primes $p, q$. Also, if $r$ is any prime dividing $e$, we have that $(p, r)=(q, r)=1$, and $\mathfrak{A}_{p} \mathfrak{A}_{q} \mathfrak{A}_{r} \leqq \mathfrak{U}_{1} \mathfrak{Y}$. Put $A_{n}=C(q, r)^{n}$ : by a theorem of Gaschutz [3], $A_{n}$ has a faithful irreducible representation over $G F(p)$. Let $M_{n}$ be a vector space over $G F(p)$ on which $A_{n}$ acts faithfully and irreducibly as a group of linear transformations. Put $B_{n}=M_{n} A_{n}$, the split extension of $M_{n}$ by $A_{n}:$ observe that $B_{n} \in \mathfrak{A}_{p} \mathfrak{A}_{q} \mathfrak{A}_{r}$. Then $M_{n}$ is the unique minimal normal subgroup of $B_{n}$, and the minimal number of generators of $M_{n}$ is at least $2^{n}$. Hence, using Lemma 2.1 (f), there is an integer $k$ such that $B_{k} \notin \mathfrak{U} \cup \mathfrak{B}: U\left(B_{k}\right)=M_{k}, V\left(B_{k}\right) \geqq M_{k}$, for $B_{k} / M_{k} \in \mathfrak{U}, B_{k} \notin \mathfrak{B}$, and $M_{k}$ is the unique minimal normal subgroup of $B_{k}$.

Suppose that $V\left(B_{k}\right)>M_{k}$ : then $C(q, r) \notin \mathfrak{B}$, but $\mathfrak{A}_{r} \leqq \mathfrak{B}$, and so $V\left(B_{k}\right)=M_{k} A_{k}^{\prime}$. But then $\left[U\left(B_{k}\right), V\left(B_{k}\right)\right] \neq 1$. However, $U_{1}\left(Y\left(B_{k}\right)\right)=1$, and so $\left[U_{1}\left(Y\left(B_{k}\right)\right), V_{1}\left(Y\left(B_{k}\right)\right)\right]=1$, contradicting 3.6.

Hence $V\left(B_{k}\right)=M_{k}$. If $\left|M_{k}\right|=p^{t}$, then $t \geqq 2$ : let $F$ be the free group 
of rank $t$ of $Q_{p}$. On $F / \zeta(F)$, which is elementary abelian of order $p^{t}$, define the action of $A_{k}$ by its action on $M_{k}$, and extend this action to give an automorphism group of $F$ : put $D=F A_{k}$.

Then $V(D)=U(D)=F$, and so $[V(D), U(D)] \neq 1$. But

$$
U_{1}(Y(D)) \leqq \zeta(F) \text {, and } V_{1}(Y(D)) \leqq F,
$$

giving

$$
\left[U_{1}(Y(D)), V_{1}(Y(D))\right]=1,
$$

contradicting 3.6 again.

Thus $\mathfrak{U}_{1}$ and similarly $\mathfrak{B}_{1}$ are nilpotent.

$$
3.8 \mathfrak{U}=\mathfrak{U}_{1} \mathfrak{Y}, \mathfrak{B}=\mathfrak{B}_{1} \mathfrak{Y} .
$$

Proof. Suppose that $\mathfrak{U}<\mathfrak{U}_{1} \mathfrak{Y}$. Then there is a group $A$ of minimal order such that $A \in \mathfrak{U}_{1} \mathfrak{Y}, A \notin \mathfrak{U}: A$ is critical, and $U(A)=\sigma A$. There are two cases to consider.

(i) $V(A)=1$. Then let $p$ be a prime which does not divide the exponent of $\mathfrak{U} \cup \mathfrak{B}$. By the theorem of Gaschutz [3], $A$ has a faithful irreducible representation over $G F(p)$. Let $M$ be a vector space over $G F(p)$ on which $A$ acts faithfully and irreducibly as a group of linear transformations, and put $B=M A$, the split extension of $M$ by $A$. Then

and so

$$
U_{1}(Y(B))=V_{1}(Y(B))=M,
$$

$$
\left[U_{1}(Y(B)), V_{1}(Y(B))\right]=1 .
$$

But $U(B)=M \sigma A$, and $V(B)=M$ : since the centralizer of $M$ in $B$ is $M$, $[U(B), V(B)] \neq 1$, contradicting 3.6.

(ii) $V(A) \neq 1$. Observe that $Y(A)$ is nilpotent and

$$
(|A / Y(A)|,|Y(A)|)=1:
$$

since $A$ is critical, $Y(A)$ is a $p$-group for some prime $p$, and is the Fitting subgroup of $A$ (that is, the maximal normal nilpotent subgroup of $A$ ), and so $\sigma A \leqq \zeta(Y(A))$. Also, since $\mathfrak{Y} \leqq \mathfrak{B}$, we have $\sigma A \leqq V(A) \leqq Y(A)$.

Now, let $A_{1}$ be isomorphic to the direct product of two copies of $A$. Let $F$ be a free group of finite rank with a normal subgroup $N$ such that $F / N \cong A_{1}$. Then if $M / N=U(F / N), M / N$ is a non-cyclic elementary abelian p-group. Also, put $Y / N=Y(F / N)$. As in Lemma 2.7, consider $Q_{p}(M):$ let $F_{1}=F / Q_{p}(M), N_{1}=N / Q_{p}(M), M_{1}=M / Q_{p}(M), Y_{1}=Y / Q_{p}(M)$. Then $M_{1} /\left[M_{1}, N_{1}\right]$ is non-abelian. Further, it is easy to deduce from the fact that $M_{1} / N_{1}$ is central in $Y_{1} / N_{1}$, and $M_{1} /\left[M_{1}, N_{1}\right]$ is nilpotent of class 2 that $N_{1} /\left[M_{1}, N_{1}\right]$ is central in $Y_{1} /\left[M_{1}, N_{1}\right]$. Then we have that 
but

$$
F_{1} /\left[M_{1}, N_{1}\right] \in\left[\mathfrak{U}_{1} \mathfrak{Y}, \mathfrak{V}_{1} \mathfrak{Y}\right]
$$

$$
F_{1} /\left[M_{1}, N_{1}\right] \notin[\mathfrak{U}, \mathfrak{B}]
$$

again contradicting $\mathbf{3 . 6}$.

The proof of $\mathbf{3 . 8}$, and with it the proof of Theorem $\mathbf{1}$, is now finished.

\section{Acknowledgement}

I would like to thank the referee for several pertinent and helpful comments about the presentation of this paper.

\section{References}

[1] Gilbert Baumslag, 'Some subgroup theorems for free v-groups', Trans. Amer. Math. Soc. 108 (1963), 516-525.

[2] John Cossey, 'Laws in nilpotent-by-finite groups', Proc. Amer. Math. Soc. (to appear).

[3] W. Gaschutz, 'Endliche Gruppen mit treuen absolut-irreduziblen Darstellungen', Math. Nachr. 12 (1954), 253-255.

[4] P. Hall, 'Finite-by-nilpotent groups', Proc. Cambridge Phil. Soc. 52 (1956), 611-616.

[5] P. Hall, 'On the finiteness of certain soluble groups', Proc. London Math. Soc. Ser. 3. 9 (1959), 595-622.

[6] Hans Liebeck, 'Concerning nilpotent wreath products', Proc. Cambridge Phil. Soc. 58 (1962), $443-451$.

[7] Hanna Neumann, Varieties of groups. Ergebnisse der Mathematik und ihrer Grenzgebiete, Bd 37, Springer, Berlin, 1967.

[8] B. H. Neumann, 'Identical relations in groups I'. Math. Ann. 114 (1937), 506-525.

[9] M. F. Newman, 'On a class of nilpotent groups'. Proc.. London Math. Soc. 3. 10 (1960), $365-375$.

[10] M. F. Newman, 'On a class of metabelian groups', Proc London Math. Soc. 3. 10 (1960), $354-364$.

[11] L. Redei, 'Die endlichen einstufig nichtnilpotenten Gruppen', Publ. Math. Debrecen 4 (1956), $130-138$.

Graduate Center

The City University of New York

New York, U.S.A. 